\title{
Sonntag für alle
}

- Rechtsvergleichende Anmerkungen zur verfassungsrechtlichen Rechtsprechung zum gesetzlichen Sonntagsschutz -

\section{Einleitung}

Rechtsvergleich, hat Günter Frankenberg einmal geschrieben, sei ein bisschen wie Reisen: er biete die Chance neuer Erkenntnisse sowohl über die Fremde als auch über die Heimat. Er berge aber eben wie das Reisen auch die Gefahr, in einer dichotomischen Betrachtungsweise zu verbleiben, die beim bloßen Abfahren von Sehenswürdigkeiten alle Phänomene entweder als ,,wie zu Hause“ oder als ,anders als zu Hause“wahrnimmt, und der so ein tieferes Verständnis sowohl des Eigenen als auch des Fremden entgeht, weil eine Reflexion über die eigene Wahrnehmungsperspektive anhand der Konfrontation mit einer fremden versäumt wird. ${ }^{1}$

Die vergleichende Analyse verfassungsrechtlicher Rechtsprechung (comparative constitutionalism) ist einer solchen Reflexion unterschiedlicher Perspektiven besonders zugeneigt; der Blick auf die Rechtsprechung unterschiedlicher Länder zum selben Themenkreis bietet in besonderem Maße die Möglichkeit einer Erkenntnis über unterschiedliche Perspektiven. Denn die Entscheidungen unterschiedlicher Verfassungsgerichte zum selben Thema stehen nicht selten in deutlichem Gegensatz zueinander. Gegensatz allerdings nicht in dem Sinne, dass sie notwendig zu unterschiedlichen Ergebnissen kommen - so wie etwa der Gesetzgeber des einen Landes zulässt, was im Gegensatz dazu derjenige eines anderen verbietet. Die Entscheidungen unterschiedlicher Verfassungsgerichte stehen nicht selten in deutlichem Gegensatz zueinander in dem Sinne, dass sie dasselbe Thema, dasselbe gesellschaftspolitische Konfliktfeld von gegensätzlichen Standpunkten aus betrachten.

Die Entscheidungen wirken dabei, nebeneinander gestellt, zuweilen wie Schuss und Gegenschuss im Film: in letzterem wird der Aufnahmestandpunkt des anderen sichtbar. Was die Perspektive des einen Bildes vernachlässigt, wird im jeweils anderen ins Licht gerückt.

Exemplarisch für solche geradezu komplementären Perspektiven sind etwa die Abtreibungsentscheidungen des U.S. Supreme Court ${ }^{2}$ und des Bundesverfassungsgerichts. ${ }^{3}$ Beim oberflächlichen sightseeing des US-amerikanischen Rechts (vor dem

1 Frankenberg, Critical Comparisons: Re-Thinking Comparative Law, Harvard International Law Journal 26 (1985), S. 411 ff, S. 411.

2 Neben Einzelfragen betreffenden Entscheidungen vor allem Roe v. Wade, 410 U.S. 113 (1973), sowie deren Nachfolgerin Planned Parenthood of Southeastern Pennsylvania v. Casey, 505 U.S. 833 (1992). Die zitierten Entscheidungen des U.S. Supreme Court sind, soweit nicht anders angegeben, unter http://www.findlaw.com/casecode/supreme.html abrufbar. Alle angegebenen Internetseiten wurden am 22. März 2010 zum letzten Mal aufgerufen.

3 BVerfGE 39, 1 (Schwangerschaftsabbruch I) und BVerfGE 88, 203 (Schwangerschaftsabbruch II). 
Frankenberg warnt) ${ }^{4}$ würde man unter Umständen nur erkennen, dass dort ,wie bei uns“ die Abtreibung in den ersten zwölf Schwangerschaftswochen straffrei möglich ist. Wer aber nur das nach Hause schreibt, der hat etwas Wesentliches verpasst. Ein Blick auf die genannten Entscheidungen der Gerichte eröffnet nämlich vollständig neue Perspektiven: Denn während der U.S. Supreme Court die Verfassungsmäßigkeit der Verurteilung der Klägerin aufgrund eines gesetzlichen Abtreibungsverbotes des Bundesstaates Texas in Roe v. Wade im Hinblick auf das Selbstbestimmungsrecht der Mutter (right of privacy) überprüft, fragt das Bundesverfassungsgericht danach, ob die durch die gesetzgeberischen Reformen der $\S \S 218 \mathrm{ff}$. StGB erfolgte, an bestimmte Umstände geknüpfte Freistellung eines Schwangerschaftsabbruchs in den ersten drei Monaten, den Anspruch des Fötus auf staatlichen Schutz seines Lebens verletzt. Das Ergebnis für die Rechtsordnungen Deutschlands und der USA - die Legalität der Abtreibung im ersten Trimester - ist also aus Sicht des jeweiligen Verfassungsrechts nicht dasselbe. Es ist einmal das gerade noch vertretbare Maximum an Schutzlosstellung ungeborenen Lebens; und es ist einmal das jedenfalls erforderliche Minimum an Entscheidungsfreiheit, das schwangeren Frauen zu gewähren ist. Reproductive autonomy als status negativus einerseits und pränataler Lebensschutz als status positivus andererseits. ${ }^{5}$

Der Wert eines solchen Vergleichs verfassungsrechtlicher Rechtsprechung ist daher, wie Donald P. Kommers treffend schreibt,

„, not so much to find the right answer to a constitutional problem, but rather to understand and distinguish the competing conceptions of freedom and responsibility that undergird the [...] cases. "6

Dies fordere dazu heraus, geschätzte Prämissen zu hinterfragen, und sei außerdem

,enlightening because the opinions and insights of foreign case law uncover truths about our own constitutional tradition that we may have only dimly perceived in the past. "7

$$
* * *
$$

4 Frankenberg (Fn. 1).

5 Eine Gegenüberstellung dieser beiden Rechtssprechungen zur Legalität von Abtreibung ist angesichts dieser verblüffenden Gegensätzlichkeit wenig überraschend - ein Klassiker des Verfassungsrechtsvergleichs, nach Neumann sogar das ,showpiece of comparative constitutional law" schlechthin, ders., Casey in the Mirror: Abortion, Abuse and The Right to Protection in the United States and Germany, American Journal of Comparative Law 43 (1995), S. 273 ff., S. 273. Im Lehrbuchklassiker Jackson / Tushnet, Comparative Constitutional Law, 1999, wird diesen Urteilen sogar das erste Kapitel gewidmet, als Einstieg und Einführung in den Verfassungsrechtsvergleich. Es überrascht ebenso wenig, dass sich die vergleichende Literatur zu diesen Urteilen angesichts dieser Gegensätzlichkeit häufig der Frage widmet, was die eine Seite von der anderen lernen kann: so etwa Neumann, a.a.O., der angesichts der Karlsruher Entscheidungen der Verbürgung staatlicher Schutzverpflichtungen im US-amerikanischen Verfassungsrecht nachgeht, oder Kommers, The Constitutional Law of Abortion in Germany: Should Americans Pay Attention?, Journal of Contemporary Health Law and Policy 10 (1993), S. 1 ff., der die Entscheidungen eher im Hinblick auf den institutionellen Kontext und die politische Kultur der beiden Länder vergleicht.

6 Kommers (Fn. 5), S. 1 - 3.

7 Ebd. 
Auch das Urteil des Bundesverfassungsgericht vom 1. Dezember $2009^{8}$ zur Verfassungsmäßigkeit des Berliner Ladenschlussgesetzes (im Folgenden: das Sonntagsurteil) hat seine transatlantischen Gegenstücke in der verfassungsrechtlichen Rechtsprechung des U.S. Supreme Court, der schon mehrfach, in einer ganzen Reihe von Urteilen zur Verfassungsmäßigkeit gesetzlichen Sonntagsschutzes Stellung genommen hat. ${ }^{9}$ Die Kernaussagen und der verfassungsrechtsdogmatische Hintergrund dieser Entscheidungen sollen zum Vergleich mit dem Sonntagsurteil im Folgenden näher betrachtet werden.

Den Urteilen ist strukturell eines gemeinsam: während das Sonntagsurteil darum kreist, wie weit der Gesetzgeber den (verfassungsrechtlich gebotenen) Schutz des Sonntags aufgeben darf, befindet sich der gesetzliche Sonntagsschutz in den Urteilen des U.S. Supreme Court - wiederum in verblüffender Spiegelbildlichkeit zur Karlsruher Entscheidung - sämtlich in der Defensive: Unter dem US-amerikanischen Verfassungsrecht muss nicht der Sonntagsschutz geschützt werden, sondern er muss sich rechtfertigen.

So sieht sich der gesetzliche Sonntagsschutz namentlich den Vorwürfen ausgesetzt, gegen das Gebot der Trennung von Staat und Religion zu verstoßen, und zum anderen die Religionsausübung unzulässig weit zu beschränken. ${ }^{10}$ Während das Bundesverfas-

81 BvR 2857/07, Juristen Zeitung 2010, S. 137 ff. (mit Anm. Classen).

9 Dabei handelt es sich um die Urteile McGowan v. State of Maryland, 366 U.S. 420 (1961), Two Guys v. McGinley, 366 U.S. 582 (1961), Braunfeld v. Brown, 366 U.S. 599 (1961), und Gallagher v. Crown Kosher Market, 366 U.S. 617 (1961). Indirekt wird der Sonntagsschutz auch in Sherbert v. Verner, 374 U.S. 398 (1963), und Estate of Thornton v. Caldor, Inc., 472 U.S. 703 (1985) verhandelt. Verfassungsrechtliche Rechtssprechung zum gesetzlichen Sonntagsschutz gibt es darüber hinaus vom Supreme Court of Canada (insbesondere: R. v. Big M Drug Mart Ltd., [1985] 1 S.C.R. 295, und R. v. Edwards Books and Art Ltd., [1986] 2 S.C.R. 713, beide online abrufbar unter http://csc.lexum.umontreal.ca/en) und dem Constitutional Court of South Africa (State v. Lawrence, State v. Negal, State v. Solberg, [1997] ZACC 11, verfügbar unter http://www.saflii.org/za/cases/ZACC/1997/11.html). Im Zentrum der vorliegende Darstellung soll - zum Zwecke einer möglichst klaren Gegenüberstellung - der Vergleich des Sonntagsurteils mit den US-amerikanischen Entscheidungen stehen. Die kanadischen und südafrikanischen Entscheidungen werden lediglich am Rande hinzugezogen. Sie sind allerdings aus vergleichender Perspektive von besonderem Interesse, weil sich die Urteile der englischsprachigen Gerichte mit den jeweils älteren intensiv auseinander setzen. So nimmt der Supreme Court of Canada auf die genannten US-amerikanischen Entscheidungen Bezug, R. v. Big M Drug Mart Ltd., a.a.O., Rd. 73 ff., und R. v. Edwards Books and Art Ltd., a.a.O., Rd. $85 \mathrm{ff}$, der Constitutional Court of South Africa sowohl auf diese wie auch auf die Kanadischen Entscheidungen, State v. Lawrence, State v. Negal, State v. Solberg, a.a.O., Rd. 98 und passim.

10 Die folgende Betrachtung beschränkt sich auf diese beiden religionsverfassungsrechtlichen Aspekte. Der Vollständigkeit halber sei aber erwähnt, dass sich die ausgewählten Urteile nicht in diesen beiden Fragen erschöpfen. Es treten in den Urteilen noch jedenfalls drei weitere verfassungsrechtliche Fragen hinzu. Insbesondere in der Entscheidung McGowan v. Maryland (Fn. 9) prüft der U.S. Supreme Court auch, ob das dort gegenständliche Gesetz bzw. dessen Ausnahmeregelungen dem Gleichbehandlungsgebot (equal protection) und der verfassungsrechtlich erforderlichen Bestimmtheit genügen. Beide Aspekte weisen allerdings kaum über den entschiedenen Einzelfall hinaus, bieten daher auch für den angestrebten Vergleich wenig Stoff. Auch in der bereits vorhandenen Literatur wird das Urteil, soweit ersichtlich, ausschließlich wegen seines religionsverfassungsrechtlichen Gehalts zitiert. Hinzutritt 
sungsgericht urteilt, dass es gesetzlichen Sonntagsschutz zum Schutze der Religionsfreiheit geben muss, so urteilt der U.S. Supreme Court, dass es gesetzlichen Sonntagsschutz auch angesichts der Trennung zwischen Staat und Religion und des Schutzes der Religionsfreiheit geben darf. Wollte man die angeführte Rechtsprechung schon an dieser Stelle auf einen ersten Blick hin zusammenfassen, so gibt es in Deutschland aus verfassungsrechtlicher Sicht den gesetzlichen Sonntag wegen des christlichen Sonntags und in den USA einen gesetzlichen Sonntag trotz des christlichen Sonntags.

\section{Ein freier Sonntag ist das Beste für alle! Die Schutzzwecke des Sonntagsschutzes in McGowan}

Gegenstand des Verfahrens in McGowan ${ }^{11}$ war eine Reihe gesetzlicher Bestimmungen des Bundesstaates Maryland, die - als so genannte Sunday closing laws, oft auch blue laws genannt - unter Androhung von Geldstrafen Vorschriften zum Schutz des Sonntags als arbeitsfreiem Tag enthielten. Diese Vorschriften bestimmten im Grundsatz das Verbot jeglicher Arbeits- und Geschäftstätigkeit am Sonntag, darunter auch, wie im deutschen Ladenschlussrecht, den Grundsatz, dass der Verkauf von Waren am Sonntag zu unterbleiben hat. ${ }^{12}$ Maryland hatte zudem (insoweit ohne zeitgenössische Entsprechung im deutschen Recht) das Öffnen und Besuchen bestimmter Vergnügungseinrichtungen („dancing saloon, opera house, bowling alley or barber shop“) ${ }^{13}$ verboten. Ausgestaltet waren alle diese Verbote durch einen schwer zu überschauenden, den deutschen Gesetzen in nichts nachstehenden Wust an Ausnahmeregelungen für bestimmte Waren oder Dienstleistungen oder bestimmte counties des Staates.

Den Klägern des Verfahrens - allesamt Beschäftigte eines Warenhauses - wurde der sonntägliche Verkauf von nicht vom Verkaufsverbot ausgenommenen Waren vorgeworfen. Der U.S. Supreme Court nahm dies zum Anlass, die Gesamtheit von Marylands Sunday closing laws auf den verfassungsrechtlichen Prüfstand zu stellen:

„Congress shall make no law respecting an establishment of religion, or prohibiting the free exercise thereof",

schließlich die Frage, ob ein gesetzlicher Ladenschluss am Sonntag nicht wirtschaftliche Grundfreiheiten (Berufs- bzw. Gewerbefreiheit, Eigentum) verletzt; dies wird insbesondere vom Constitutional Court of South Africa in State v. Lawrence, State v. Negal, State v. Solberg (Fn. 9) ausführlich erörtert.

11 Die den Entscheidungen von McGowan v. Maryland und Two Guys v. McGinley zugrunde liegenden Fälle waren „essentially the same“, und der U.S. Supreme Court behält seine wesentlichen Erörterungen daher der Entscheidung McGowan vor, siehe Two Guys v. McGinley (Fn. 9), 584.

12 Auch der deutsche gesetzliche Sonntagsschutz ist natürlich nicht auf den (seit der Föderalismusreform I nunmehr landesrechtlich geregelten) Ladenschluss beschränkt. Zentrale Vorschriften des Sonntagschutzes sind vielmehr die über den Handel weit hinausgehenden $\S \S 9$ ff. Arbeitszeitgesetz. Für eine Übersicht über die einfachgesetzlichen Sonntagsschutzvorschriften quer durch die Rechtsgebiete siehe Korioth in Maunz/Dürig, Grundgesetz, Art. 139 WRV (42. Ergänzungslieferung 2003), Rn. 54 ff.

13 McGowan v. Maryland (Fn. 9), S. 423. 
lautet der erste Halbsatz des First Amendments, und dieser bildet die Textgrundlage des US-amerikanischen Religionsverfassungsrechts. ${ }^{14}$ Auf ihr ruhen die beiden wesentlichen dogmatischen Kategorien zur Bestimmung des Verhältnisses zwischen Staat und Religion, auf dem Verbot eines gesetzlichen establishment of religion einerseits und der Garantie eines free exercise von Religion andererseits. ${ }^{15}$

14 Die Gewährleistungen des First Amendments verpflichten über dessen Wortlaut hinaus nicht nur den Bundesgesetzgeber („Congress shall make no law..."), sondern auch die Bundesstaaten. Denn die wesentlichen Gewährleistungen der Bill of Rights (der ersten zehn Amendments) sollen durch den due-process-clause des $14^{\text {th }}$ Amendment ,inkorporiert", d.h. auf die Staaten erstreckt worden sein. Grundlegend insoweit Duncan v. Louisiana, 391 U.S. 145. Zu dieser (etwas verschlungenen) ,,incorporation controversy“ siehe Sullivan / Gunter, Constitutional Law, 15. Aufl., 2004, S. 467 ff. Wie Dreisbach allerdings zurecht erinnert, ist das First Amendment ,neither the only nor the first constitutional measure that addressed matters of religion and politics“, ders., Constitution's Forgotten Religion Clause: Reflections on the Article VI Religious Test Ban, Journal of Church and State 38 (1996), S. 261 ff, S. 261. Dieser no-religious-test-clause in Art. VI Satz 3 lautet: , [...] no religious Test shall ever be required as a Qualification to any Office or public Trust under the United States“. Die Bestimmung hat tatsächlich in der religionsverfassungsrechtlichen Rechtsprechung kaum Spuren hinterlassen, und findet daher wohl zurecht auch in den meisten Standardwerken zum US-amerikanischen Verfassungsrecht kaum oder keinerlei Erwähnung. Auch Dreisbach selber gelangt in seiner rechtshistorischen Untersuchung zu dem Ergebnis, die Bestimmung sei eher in föderaler denn in religionsverfassungsrechtlicher Hinsicht von Bedeutung, Dreisbach, a.a.O., 293 ff; dem zustimmend: Walter, Religionsverfassungsrecht, 2006, S. 77 ff.

15 Die Versuchung ist groß, den Trennungsgrundsatz des no establishment als den ,institutionellen" oder organisationsrechtlichen Teil des US-amerikanischen Religionsverfassungsrecht $\mathrm{zu}$ lesen und als solchen der grundrechtlichen, subjektivrechtlichen Gewährleistung des free exercise gegenüberzustellen. Als sei no establishment Staatskirchenrecht, free exercise Religionsausübungsfreiheit im Sinne unseres Verfassungsrechts. Doch eine solche Systematisierung geht nicht auf. Und spätestens nach der „Vergrundrechtlichung“ des Art. 139 WRV durch das Sonntagsurteil greift eine solche Klassifizierung in institutionelles und subjektivrechtliches Religionsverfassungsrecht ja auch für das deutsche Verfassungsrecht kaum mehr. In der US-amerikanischen Standardliteratur wird no establishment durchweg als Teil der Bill of Rights unter dem Rubrum freedom of religion im Grundrechtsteil der Lehrbücher behandelt, ohne dass dem erkennbar eine bewusste materiellrechtlich-dogmatische Einordnung zugrunde läge, vgl. Rotunda / Nowak, Treatise on Constitutional Law - Substance and Procedure, 2. Aufl. 1992, Bd. IV, S. 453 ff.; dies., Constitutional Law, 6. Aufl. 2000, S. 1311 ff.; Stone u.a., Constitutional Law, 6. Aufl. 2009, S. 1501 ff.; Sullivan / Gunter (Fn. 14), S. 1503 ff.; Barron / Dienes, Constitutional Law in a Nutshell, 6. Aufl. 2005, S. 546 ff. Auch der U.S. Supreme Court seinerseits hat no establishment nie ausdrücklich als ein Grundrecht klassifiziert. Er spricht dem Grundsatz aber dennoch eindeutig subjektivrechtliche Qualität $\mathrm{zu}$, indem er prozessual dem einzelnen Normadressaten standing, d.h. das Recht, Klage zu erheben, zuerkennt. Das Gericht begründet dies (unter Fortsetzung seiner bisherigen Rechtsprechung) damit, die Trennung von Staat und Kirche diene im Kern gerade der Sicherung individueller Freiheit (McGowan v. Maryland (Fn. 9), S. 430). Das Gericht beruft sich dabei, wie bereits in früheren Urteilen, auf Madison, dem „Architekten des First Amendment“, konkret auf dessen berühmtes Pamphlet „Memorial and Remonstrance Against Religious Assessments" von 1785, verfasst im Rahmen seiner (letztlich erfolgreichen) Kampagne gegen Bestrebungen in der Virginia Assembly zur Einführung einer Kirchensteuer, die der heutigen in Deutschland gar nicht wenig ähnelte. Die Sponsoren des Gesetzesvorhabens hofften, dem vermeintlichen moralischen Verfall des Nachkriegsamerika durch finanzielle Besserstellung der Kirchen begegnen zu können, Blasi, School Vouchers and Religious Liberty: Seven 
In McGowan geht es um den ersten Grundsatz: errichtet der Staat, indem er den Sonntag zum arbeitsfreien Tag für alle erklärt, ein religious establishment, wird so eine religiöse Einrichtung, der christliche Sonntag, zur staatlich-religiösen Einrichtung? Der U.S. Supreme Court verneint dies im Ergebnis: der Sonntagsschutz sei eine weltliche Angelegenheit, der Gesetzgeber mithin befugt, ihn zu verfügen.

Der Kern der Erörterung des U.S. Supreme Court besteht in einer Analyse der Funktion, der Zwecke der Sunday legislation, der historischen und der zeitgenössischen Zielsetzung des Gesetzgebers. In aller Breite ${ }^{16}$ schildert das Gericht die Entwicklung des gesetzlichen Sonntagsschutzes und die ihn begleitende gesellschaftliche und juristische Diskussion von dem Verbot Heinrichs III. aus dem Jahre 1237, sonntags Markt zu halten, bis zu den zum Zeitpunkt des Urteils, im Jahr 1961, noch in allen Bundesstaaten bestehenden Sunday closing laws.

Das Gericht kommt dabei zu dem Ergebnis, dass diese Gesetzgebung zwar ursprünglich unzweifelhaft dazu bestimmt war, den christlichen Sonntag zu sanktionieren und den Kirchgang der Bevölkerung zu fördern, aber heute nicht mehr wesentlich religiös motiviert sei, sondern vielmehr säkularen sozialpolitischen Zwecken verpflichtet sei. In der historischen Linie, die die Richter ziehen, ist der Sonntag so zwar über die Jahrhunderte unverändert als arbeitsfrei bestimmt worden, doch die Gründe hierfür sind mit der Zeit ausgetauscht worden. Sunday closing laws seien vielmehr integraler Bestandteil moderner Wohlfahrtsgesetzgebung, die sich öffentlicher Gesundheitsvorsorge, den Arbeitsbedingungen für Frauen und Kinder, der Arbeitssicherheit und der Arbeitszeitbe-

Questions from Madison's Memorial and Remonstrance, Cornell Law Review 87 (2001-2002), S. 783. Bei Madison, erbitterter Gegner eines solchen Vorhabens, heißt es an der entscheidenden Stelle: „What influence in fact have ecclesiastical establishments had on Civil Society? In some instances they have been seen to erect a spiritual tyranny on the ruins of the Civil authority; in many instances they have been seen upholding the thrones of political tyranny: in no instance have they been seen the guardians of the liberties of the people", Madison, A Memorial and Remonstrance, 1785, abgedruckt bei Blasi (Hrsg.), Ideas of the First Amendment, 2006, S. 162ff., S. 165. Eine Aussage, die man als recht barsche Absage an das deutsche Kooperationsmodell des Staatskirchenrechts verstehen muss. Interessanterweise argumentiert Madison in „Memorial and Remonstrance“ allerdings nicht nur ,republikanisch" freiheitswahrend gegen die Gefahren eines Staates, der Gesinnungen fördert. Er verbindet diese Warnung mit einer weiteren, viel seltener zitierten, aber um nichts weniger bemerkenswerten: Eine Vermengung zwischen Staat und Religion ist nach seiner Ansicht nämlich nicht nur geeignet, das Gemeinwesen zur Tyrannei werden zu lassen. Eine solche Vermengung ist gleichermaßen geeignet, Religion und Religionsgemeinschaften zu korrumpieren! Die Trennung von Staat und Kirche ist für ihn daher auch um der Religion selbst willen geboten ,[because] experience witnesseth that ecclesiastical establishments, instead of maintaining the purity and efficacy of Religion, have had a contrary operation. During almost fifteen centuries has the legal establishment of Christianity been on trial. What have been its fruits? More or less in all places, pride and indolence in the Clergy, ignorance and servility in the laity, in both, superstition, bigotry and persecution." (Madison, a.a.O, S. 164). Die Madisonsche Konzeption ist damit sowohl darauf ausgerichtet, den Staat vor der Religion als auch die Religion vor dem Staate zu schützen; und sie weist insofern vielleicht überraschenderweise eine tiefer liegende Verbundenheit mit dem zunächst ganz entgegengesetzt wirkenden, vom Gedanken kirchlicher Selbstbestimmung getragenen Modell deutschen Staatskirchenrechts auf.

16 McGowan v. Maryland (Fn. 9), S. 431 ff. 
grenzung widme, oder auch Parks und Strände und kulturelle Einrichtungen zur Verfügung stelle, kurz: die den Weg weise „,toward the good life for all““.17

Sonntagsschutzgesetze wegen ihrer religiösen Provenienz für unzulässig zu erklären, hieße somit dem Gesetzgeber zu verbieten, die public welfare zu fördern - mit der Trennung von Kirche und Staat habe dies indes nichts mehr zu tun. ${ }^{18}$ Die vielen Ausnahmen, die die angegriffene Maryland statute vom Grundsatz der Arbeitsfreiheit zulasse - Ausnahmen, die deutlich über die nach religiösem Maßstab zulässigen notwendigen oder wohltätigen Beschäftigungen hinausgehen - zeigten das Bestreben,

,to set one day apart from all others as a day of rest, repose, recreation and tranquility - a day which all members of the family and community have the opportunity to spend and enjoy together, a day on which there exists relative quiet and disassociation from the everyday intensity of commercial activities, a day on which people may visit friends and relatives who are not available during working days. "19

Man kann berechtigte Zweifel an dem Indizienbeweis der Religionsferne der streitgegenständlichen bundesstaatlichen Gesetze durch den U.S. Supreme Court haben. Immerhin bedienen sich die Gesetze Marylands einer augenfällig religiös konnotierten Sprache, die dem deutschen (technisch-verwaltungsrechtlich gefassten) Ladenschlussgesetzen gänzlich fremd ist. So heißt es etwa einleitend in Art. 27, § 492 Maryland Annotated Code unter der Überschrift „Sabbath Breaking“(!): „No person whatsoever shall work or do any bodily labor on the Lord's day, commonly called Sunday", und weiter wird es untersagt, ,to profane the Lord's day by gaming, fishing, fowling, hunting““ ${ }^{20}$ Formuliert so ein weltlich-sozialpolitisch motivierter Gesetzgeber? Auch in der Sache verwundern die Verbote: sind nicht gerade Angeln und Kegeln und Tanzen die Unternehmungen schlechthin, bei denen Familien und Freunde zusammenfinden? Ein solcher Profanisierungsschutz des Sonntages ist jedenfalls im deutschen Sonntagsschutzrecht nicht zu finden, das aber gerade - im Gegensatz zum US-amerikanischen - eine die christliche Religion schützende Institution sein soll. Verteidigt der U.S. Supreme Court eine ganz religiös daherkommende Gesetzgebung als weltliche, das Bundesverfassungsgericht eine ganz und gar nüchtern-weltliche als religiöse?

An eben dieser Frage - nach den Zwecken des Sonntagsschutzes - scheiden sich im Übrigen die Wege des U.S. Supreme Court und des Supreme Court of Canada. Auch letzterer fragt sich in Big M Drug Mart ${ }^{21}$ im Hinblick auf eine mögliche Verletzung der Religions- und Gewissensfreiheit aus Sec. 2 (a) der Canadian Charter of Rights and Freedoms, ob der angegriffene Lord's Day Act religiöse oder weltliche Ziele verfolgt. Eben wie der U.S. Supreme Court steht auch für das kanadische Gericht außer Frage, dass das Gesetz ursprünglich dem religiösen Zweck, die Sonntagsobservanz zu fördern, dienen sollte. Die Vorstellung aber, das Gesetz könne inzwischen seine Zielsetzung gewandelt haben, und wegen seiner nunmehr säkularen Finalität verfassungsrechtlich unbedenklich sein, lehnt das Gericht (unter ausdrücklicher Absetzung von McGowan

17 McGowan v. Maryland (Fn. 9), S. 445.

18 McGowan v. Maryland (Fn. 9), S. 445.

19 McGowan v. Maryland (Fn. 9), S. 450.

20 McGowan v. Maryland (Fn. 9), S. 453 (Hervorhebungen von mir).

21 R. v. Big M Drug Mart Ltd. (Fn. 9), Rd. 78 ff. 
und der übrigen US-amerikanischen Rechtsprechung) ab: die Annahme eines solchen „shifting purpose“ würde in praktischer Hinsicht dazu führen, dass über die Verfassungsmäßigkeit von Gesetzen immer und immer wieder gestritten werden könnte; vor allem sei als Gesetzeszweck aber immer nur die Intention des historischen Gesetzgebers zu verstehen. ${ }^{22}$ Wandeln könne sich allenfalls der Effekt eines Gesetzes:

„While the effect of such legislation as the Lord's Day Act may be more secular today than it was in 1677 or in 1906, such a finding cannot justify a conclusion that its purpose has similarly changed. In result, therefore, the Lord's Day Act must be characterized as it has always been, a law the primary purpose of which is the compulsion of sabbatical observance. "23

Auch das Bundesverfassungsgericht beschäftigt sich mit der „weltlich-sozialen“ Bedeutung der „seelischen Erhebung“, der der Sonntagsschutz als „Grundlage für die Rekreationsmöglichkeiten des Menschen und zugleich für ein soziales Zusammenleben“ diene. ${ }^{24}$ Auch das Bundesverfassungsgericht stößt unweigerlich auf die Koinzidenz des christlichen Gebotes mit dem sozialpolitisch Guten:

„,Art. 139 WRV ist damit ein religiöser, in der christlichen Tradition wurzelnder Gehalt eigen, der mit einer dezidiert sozialen, weltlich-neutral ausgerichteten Zwecksetzung einhergeht." 25

Beide Gerichte beschäftigen sich also mit dieser Koinzidenz weltlicher und religiöser Zwecke im Sonntagsschutz, und beide Gerichte scheinen im Wesentlichen zur selben sozialgeschichtlichen Erkenntnis zu gelangen: der Ursprung der Arbeitsfreiheit des Sonntags sei zwar religiös, heute aber bündelten sich im Sonntagsschutz eine Vielzahl weltlicher Überlegungen.

Aber für die beiden Gerichte verbindet sich damit jeweils eine gegenläufige Strategie: Der U.S. Supreme Court muss zum Zwecke seiner Entscheidung beweisen, dass, wenn der Sonntagsschutz auch in der christlichen Tradition wurzele, er nunmehr doch gänzlich weltlich ausgerichtet sei, um die Konsequenz eines verbotenen establishments zu vermeiden; das Bundesverfassungsgericht muss erläutern, weshalb sich der Staat ausgerechnet mit einer Institution, die so vielerlei weltliche Zwecke verknüpft, schützend vor die Religionsausübungsfreiheit der christlichen Kirchen stellt. Liest man beide Urteile nebeneinander, so fällt einem auf, wie sehr beide Urteile, jedes für sich, ihre eigene Argumentation zu unterlaufen scheinen:

22 Ebd.

23 R. v. Big M Drug Mart Ltd. (Fn. 9), Rd. 93. In der Konsequenz erklärt das Gericht das Gesetz für verfassungswidrig.

24 BVerfG (Fn. 8), Rd. 139.

25 BVerfG (Fn. 8), Rd. 141. Ganz ausdrücklich von einer Koinzidenz spricht auch Justice Frankfurter in seiner concurring opinion zu McGowan (und den Parallelentscheidungen): „Innumerable civil regulations enforce conduct which harmonizes [...] with religious canons. State prohibitions of murder, theft and adultery reinforce commands of the decalogue", McGowan v. Maryland (Fn. 9), S. 462. 
Der U.S. Supreme Court verstrickt sich erstaunlich tief in die angelsächsische, koloniale und frühe US-amerikanische Geschichte religionsfördernder Gesetzgebung bei dem Versuch zu beweisen, der gesetzgeberisch gewollte „uniform day of rest“ sei nur zufälligerweise bzw. historisch kontingenterweise ein Tag mit besonderer Bedeutung für die christliche Mehrheitsreligion. ${ }^{26}$ Das Bundesverfassungsgericht im Gegenzug bedient sich sozialwissenschaftlicher Sachverständiger und zitiert deren Erkenntnisse psychosozialer Deprivationserscheinungen durch verlängerte Arbeitszeiten und Desynchronisationseffekte (von veränderter politischer Teilhabe bis hin zur „Verringerung der sozialen Interaktionsdichte und -qualität" ${ }^{27}$ - innerhalb eines Urteils, dessen verfassungsrechtlicher Knackpunkt darin liegt zu zeigen, dass Art. 139 WRV „funktional auch auf die Inanspruchnahme und Verwirklichung des Grundrechts der Religionsfreiheit angelegt" ${ }^{28}$ ist. Eigentlich hätte der U.S. Supreme Court solche Sachverständige gebraucht, einen Brandeis brief, der mit entsprechenden Studien den Gesetzgeber in seinen vermeintlich weltlichen Zielsetzungen stützt; ${ }^{29}$ das Bundesverfassungsgericht hätte aber vielmehr erörtern müssen, inwiefern die vermeintliche „,funktionale Ausrichtung“ der Kirchenartikel auf die Religionsfreiheit auch eine inhaltliche ist. Unterläuft den beiden Spruchkörpern ein Lapsus, der tiefer blicken lässt? Bewahrt der U.S. Supreme Court vielleicht doch unter dem Deckmantel der Sozialpolitik ein Privileg der christlichen Mehrheitsreligion? Und verteidigt das Bundesverfassungsgericht vielleicht im Gegenzug unter dem Deckmantel des Schutzes der Religionsausübungsfreiheit das sozialpolitisch Wünschenswerte? Vielleicht -.

Jedenfalls verhandeln beide Urteile im Rahmen dieser Koinzidenz weltlicher und religiöser Zwecke des Ruhetages Sonntag je einen Aspekt, der dem jeweils anderen zu entgehen scheint. Die Urteile haben sich gegenseitig etwas mitzuteilen:

Da McGowan den Schutz des Sonntags als zulässigen Erlass eines „uniform day of rest" rechtfertigt, ist damit zugleich gesagt (und diese Möglichkeit wird auch ausdrücklich angesprochen), dass dieser gemeinsame Ruhetag auch jeder andere Tag der Woche

26 McGowan v. Maryland (Fn. 9), S. 444 f.

27 BVerfG (Fn. 8), Rd. 169.

28 BVerfG (Fn. 8), Rd. 138.

29 Brandeis, später selbst Richter am U.S. Supreme Court, hatte diese Form des im US-amerikanischen Verfassungsprozess ganz üblichen, nicht von Rechtsausführungen, sondern von sozialwissenschaftlichen, empirischen Daten getragenen Schriftsatzes als junger politischer Anwalt erstmals eingesetzt, um die Richter im Verfahren Muller v. State of Oregon, 208 U.S. 412 (1908), von den enormen gesundheitlichen und sozialen Gefahren überlanger Arbeitsstunden für Frauen, und damit von der Verfassungsmäßigkeit einer gesetzlichen Arbeitszeitbegrenzung für Arbeiterinnen zu überzeugen. Der Erfolg dieses legendären Brandeis brief beendete die schon von dem abweichenden Richter Holmes als sozialdarwinistisch gebrandmarkte Rechtssprechung aus Lochner v. People of State of New York, 198 U.S. 45 (1905), die jegliche Arbeitszeitgesetzgebung als unzulässige Verkürzung der Vertragsfreiheit verworfen hatte. Der Brandeis brief steht aber über seinen konkreten politischen Erfolg hinaus symbolisch für die zentrale Denkwende, die das US-amerikanische Recht im 20. Jahrhundert vollzogen hat, nämlich für die Abwendung von der Formalität des classical legal thought des 19. Jahrhunderts, das die richtige Rechtsanwendung im methodenkohärent aufgefundenen systemkonsistenten Ergebnis sah, hin zur sociological jurisprudence und zum legal realism, die die außerrechtlichen, gesellschaftlichen Folgen der Rechtsentscheidung zu deren Maßstab machten, siehe dazu Horwitz, The Transformation of American Law 1870-1960: The Crisis of Legal Orthodoxy, 1992, S. 187ff., S. 208ff. und passim. 
sein könnte. Wenn der Sonntag nämlich tatsächlich religiöse und weltliche Zwecke gleichermaßen hat, und diese weltlichen und religiösen Zwecke tatsächlich, wie der U.S. Supreme Court meint, trennscharf voneinander unterschieden werden können, ja, wie das Gericht ebenfalls meint, diese Zwecke einander historisch gesehen abgelöst haben, dann sieht der U.S. Supreme Court tatsächlich etwas ganz Einfaches, was das Bundesverfassungsgericht nicht sieht, nicht sehen kann: man könnte die unterschiedlichen Zielsetzungen an unterschiedlichen Tagen befriedigen. Man könnte den weltlichen Sonntag verlegen! Nur dass es dem Gericht unsinnig erscheint, dem Gesetzgeber einen solch ungeheuren praktischen Aufwand abzuverlangen, den arbeitsfreien Tag zu verschieben, nur um das Zusammenfallen mit dem christlichen Feiertag zu vermeiden. ${ }^{30}$ Im Rahmen von Art. 139 WRV kann diese Frage natürlich gar nicht erst auftauchen: denn dort ist ja die Verwirklichung der Zwecke eines Ruhetages (,seelische Erhebung“) bereits verbindlich auf den einen ganz bestimmten Tag der Woche festgelegt.

Aber vielleicht lassen sich weltliche und religiöse Zwecke des Sonntags gar nicht so trennscharf voneinander unterscheiden, wie es der U.S. Supreme Court gern haben möchte? Erstaunlich barsch bescheidet letzterer die Kläger in McGowan, beim Sonntag als wöchentlichem Ruhetag dürfe es doch nun wirklich bleiben, weil dies nun einmal der traditionelle Tag der Ruhe sei. ${ }^{31}$ Dieser Rekurs auf die Tradition will nicht recht hineinpassen in ein Urteil, dass das angegriffene Gesetz nur deshalb aufrecht erhält, weil sich der Sonntagsschutz von seiner religiösen Tradition emanzipiert habe: Bei einem wöchentlichen Ruhetag soll es bleiben dürfen, weil es gute nicht-religiöse Gründe für einen wöchentlichen Ruhetag gibt, weil religiöse Gründe für sein Beibehalten angeblich gar keine Rolle mehr spielen; dieser wöchentliche Ruhetag darf aber der christliche Ruhetag, von dem er abstammt, bleiben, weil - ja warum eigentlich?

Hier regt sich ein Verdacht. Dass nämlich die feine Logik des U.S. Supreme Court, beim gesetzlichen Sonntagsschutz handele es sich (inzwischen) schlicht um einen Fall moderner Sozialgesetzgebung, nicht recht aufgehen mag. Nicht nur, dass die Tradition eines wöchentlichen Ruhetages religiösen Ursprungs ist - das diskutiert das Gericht ja ausführlich. Sondern vielmehr dass der Ursprung des christlichen Sonntags, dass das religiöse Gebot eines wöchentlichen Ruhetages sehr viel mit den angeführten sozialpolitischen Gründen zu tun hat, ja durch sie motiviert sein könnte. Ein solcher Gedanke muss von einem Gericht, das die Konsequenz einer Verquickung von Staatlichem und Kirchlichem vermeiden will, tunlichst umgangen werden. Dieser Gedanke tritt aber im Sonntagsurteil deutlich zu Tage, wenn das Bundesverfassungsgericht die Interpretation des Sonntags im Selbstverständnis der Kirchen referiert. Der Sonntag diene nach deren Glauben dazu, so die Kirchen selbst, „Schutz vor einer weitgehenden Ökonomisierung des Menschen zu bieten“, „,der Sonntag unterbreche den Arbeitsalltag und gewähre eine Ruhepause“, und durch den freien Sonntag „erhalte [das Leben der Menschen] durch die Arbeit und die Ruhe seinen Rhythmus“.32 Ist die Geschichte der religiösen und der weltlichen Sonntagsruhe vielleicht eine gemeinsame?

Ein Blick in die religionsgeschichtliche Literatur scheint dies zu bestätigen. Ein solcher liefert nämlich die überraschende Erkenntnis, dass der Sonntag, wenngleich der

30 McGowan v. Maryland (Fn. 9), S. 506 ff.

31 Ebd.

32 BVerfG (Fn. 8), Rd. 143. 
wichtigste Tag der Woche, keineswegs zu allen Zeiten die zentrale Bedeutung für das Christentum hatte, wie es uns heute erscheint. Der Sonntag war vielmehr Jahrhunderte lang liturgisch so niedrigen Ranges, dass er hinter den verstreut liegenden Heiligenfesten regelrecht zurücktrat. ${ }^{33}$ Danach ist der Sonntag, auch theologisch besehen, zu einem Gutteil eine moderne Erscheinung, ja eine des 20. Jahrhunderts: so gelingt der ,entscheidende Durchbruch“ für den Sonntag in der katholischen Kirche erst mit dem Zweiten Vatikanischen Konzil, das ihn erstmals zum „Fundament und Kern des gesamten liturgischen Jahres“" erklärt. ${ }^{34}$ In praktisch-theologischer Hinsicht verweisen die Kommentatoren einheitlich auf die Bedeutung des Sonntages als Gegenpol und Durchbrechung der modernen Wirtschafts- und Arbeitswelt, ,,mit ihrer Tendenz zur sog. Rundum-die-Uhr-Gesellschaft“, ${ }^{35}$ die das Leben durchökonomisiere, und kaum noch Zeit lasse für „Sozialzeit“, für „Ruhe und Erholung“, „Gemeinschaft in Familie“ oder „Dienst am Nächsten“. ${ }^{36}$ So besehen ist der Sonntag möglicherweise nicht historisch religiös motiviert gewesen und heute auch oder zunehmend sozialpolitisch. Er ist vielleicht vielmehr historisch ein Ergebnis kirchlicher Sozialpolitik. Der moderne gesetzliche Sonntagsschutz wäre somit das Ergebnis eines (fortgesetzten) „Kampfes um den Sonntag", in dem die Kirchen (gemeinsam mit Gewerkschaften und Sozialpolitikern unterschiedlichster Prägung) mehr oder weniger erfolgreich an die christliche Tradition der sonntäglichen Ruhe anknüpfen konnten. Und in diesem Sinne zu einem guten Teil eben ein kirchlicher oder religiöser Beitrag „toward the good life for all“.37 In dem Oszillieren des Sonntagsurteils zwischen dem Religionsbezug und dem allgemeinen Nutzen des Sonntagsschutzes tritt diese innere Verwachsenheit beider Aspekte zu Tage, und entlarvt womöglich die Trennbarkeit der beiden, auf der der U.S. Supreme Court beharrt, als historischen Irrtum.

Die Koinzidenz der weltlich sozialpolitischen und der religiösen Ziele ist somit vielleicht eine historische Konvergenz? Die Koinzidenz der gottgefälligen Sonntagsruhe und dem Freizeitbedürfnis des modernen Menschen ist aber andererseits nicht ganz ohne Brüche. Denn die Befriedigung des Freizeitbedürfnisses verlangt zuweilen, dass ein anderer arbeitet. Die (verwaltungsrechtliche) Rechtsprechung zum Sonntagsschutz hatte diesen Widerspruch mit der Unterscheidung zwischen „Arbeit für den Sonntag“ und „Arbeit trotz des Sonntages“" zu lösen gesucht. Arbeit, die der Freizeitgestaltung anderer dient - Arbeit für den Sonntag anderer hätte es daher richtig heißen müssen -, konnte somit für zulässig erachtet werden. ${ }^{38}$

Zurecht ist in Reaktion auf das Sonntagsurteil darauf hingewiesen worden, dass es nicht unbedingt leichter geworden sein dürfte, diese Unterscheidung zu treffen. In die

33 Schnitker in Kasper u.a. (Hrsg.), Lexikon für Theologie und Kirche, 3. Aufl. 2000, Bd. 9, Eintrag „Sonntag“, S. 730.

34 Ebd.

35 Rinderspacher in Betz u.a. (Hrsg.), Religion in Geschichte und Gegenwart: Handwörterbuch für Theologie und Religionswissenschaft, 4. Aufl. 2004, Bd. 7, Eintrag „Sonntag“, S. 1448.

36 Schnitker (Fn. 33).

37 McGowan v. Maryland (Fn. 9), S. 445.

38 S. bspw. BVerwGE 90, 337 (Öffnung eines Bräunungsstudios am Sonntag). 
Stadt gehen und Einkaufen ist eben eine weit verbreitete Form der Freizeitgestaltung. Sie dient der Erholung und sie erfordert offene Läden. Geld ausgeben ist außerdem womöglich, wie Jens Bisky unter Berufung auf die Soziologin Eva Illouz angeführt hat, weit mehr als nur Geschäfte machen. Konsum beruhe in ganz erheblichem Maße auf „romantischen und idealistischen Impulsen“, wie etwa der Kauf einer Reise für die Familie, eines Souvenirs für einen Freund oder eines Parfüms für die eigene Anziehungskraft. ${ }^{39}$ Shopping zur seelischen Erhebung ist die Aporie des sonntäglichen Ladenschlusses. Sollten die Sphären des Intimen und der Wertschöpfung tatsächlich so unentrinnbar miteinander verschränkt sein, wie $I l l o u z^{40}$ nahe legt, dass sich erstere immer nur unter den Bedingungen der letzteren konfiguriert, wäre der Ruf der Kirchen nach sonntäglichem Schutz des Menschen vor vollständiger Ökonomisierung keiner nach menschlicher Wärme mehr, sondern einer nach sozialer Askese.

Andere wiederum leugnen nicht den akommerziellen Gehalt kommerzieller Praktiken, sondern bestreiten grundsätzlich, dass die Abstinenz von Arbeit und Geschäftigkeit überhaupt einen gesellschaftlichen Wert haben könne. So schreibt etwa Marc A. Stadtmauer recht forsch, trotz familienpolitischer Erwägungen sei es ,obvious that the best interests of society as a whole“" wohl in der Aufhebung des Sonntagsschutzes liege. ${ }^{41}$ So habe beispielsweise die Aufhebung des Ladenschlusses in Massachusetts $1983 \mathrm{zu}$ einem kleinen Boom und 6000 neuen Arbeitsplätzen geführt. ${ }^{42}$ Ohne die Bedeutung neuer Arbeitsplätze (gerade auch für Familien) zu unterschätzen, dürfte eine solche Gleichsetzung gesamtgesellschaftlichen Wohlergehens mit wirtschaftlichen Kennzahlen wohl etwas verkürzend sein. Im Übrigen sind Stadtmauers Annahmen womöglich falsch. In seiner eigenen Quelle findet sich nämlich der deutliche Hinweis darauf, dass der retail boom im Massachusetts des Jahres 1983 zu einem Großteil darauf beruht haben dürfte, dass die neue Möglichkeit des Sonntagseinkaufes zahlreiche Bewohner der Nachbarstaaten Rhode Island und New Hampshire angezogen hatte. ${ }^{43}$ Die Einzelhandelsumsätze könnten sich daher primär zwischen den Bundesstaaten verlagert haben, jenseits der Bundesstaatsgrenzen also Arbeitsplätze verloren gegangen sein. Dass längere und wochenendliche Ladenöffnung nicht zu einer Vergrößerung sondern nur zu einer Verlagerung der Einzelhandelsumsätze führe (insbesondere von den Kleinen zu den Großen) - eine im Hinblick auf die Begrenztheit eines jeden Budgets im Grunde logisch zwingende Konsequenz - war ja auch schon immer das wirtschaftspolitische

39 Bisky, Wem gehört der siebte Tag?, Süddeutsche Zeitung vom 1. Dezember 2009, S. 11; ähnliche Hinweise auch bei Göbel, Argumentationsnot, Frankfurter Allgemeine Zeitung vom 2. Dezember 2009, S. 11 und Classen, Anmerkung zu BVerfG, 1 BvR 2857/07, Juristen Zeitung 2010, S. $144 \mathrm{f}$.

40 Bisky bezieht sich auf Illouz, Der Konsum der Romantik - Liebe und die kulturellen Widersprüche des Kapitalismus, 2003, indem die Autorin die Überschneidung von romantischer Liebe mit ökonomischen Praktiken durch eine „Romantisierung der Waren und [...] Verdinglichung der romantischen Liebe“ (ebd., S. 23) beschreibt.

41 Stadtmauer, Remember the Sabbath? The New York Blue Laws and the Future of the Establishment Clause, Cardozo Arts \& Entertainment Law Journal 12 (1994), S. 213 ff., S. 235 (Hervorhebung von mir).

42 Stadtmauer (Fn. 41), Fn. 125.

43 Cooper, Sunday Shopping Architect of Blue Laws' End Reports Tax, Job Bonanza in Past Year, Boston Globe vom 24. März 1984, S. 1. 
Argument der Gegner einer Liberalisierung der Ladenöffnung. In diesem Sinne hatten auch die Gewerkschaften vor dem Bundesverfassungsgericht Stellung genommen. ${ }^{44}$

$* * *$

Es ist aber unter dem US-amerikanischen Verfassungsrecht möglicherweise das letzte Wort zum Sonntagsschutz noch nicht gesprochen. Denn die Rechtsprechung zur noestablishment-Doktrin hat sich seit den hier im Mittelpunkt stehenden Urteilen des Jahres 1961 entscheidend weiterentwickelt und ist über den reinen purpose test von McGowan weit hinaus gelangt. Die Gegner der blue laws jedenfalls sind optimistisch. ${ }^{45}$ Seit den 1970er Jahren unterwirft der U.S. Supreme Court unter dem no establishment angegriffene gesetzliche Regelungen nämlich einer dreistufigen Prüfung, fragt also erstens, wie bereits in McGowan, danach, ob die Gesetzgebung primär säkulare Zwecke verfolgt, sodann ob die Gesetzgebung sich primär säkular auswirkt und schließlich drittens, ob die Gesetzgebung zu einer übermäßigen Verflechtung (entanglement) von Staat und Religion führe. Unter diesem sog. Lemon Test ${ }^{46}$ könnte die Verfassungsmäßigkeit der Sunday closing laws tatsächlich in neuem Licht erscheinen. Denn diese förderten, so wird argumentiert, den christlichen Sonntag jedenfalls im Effekt. ${ }^{47}$

\section{Schutz vor dem Sonntagsschutz? Das Problem der religiösen Minderheiten in Braunfeld}

Wenn das Gericht in McGowan den Gesetzgeber gewähren lässt, die Mehrheit also ihre Vorstellung vom guten Leben gesetzlich verfügen darf, dann stellen Braunfeld ${ }^{48}$ und Crown Kosher Market ${ }^{49}$ die verfassungsrechtlich zwingende Anschlussfrage: Was wird mit der gesellschaftlichen Minderheit, die das (mehrheitlich) gewollte Gute nicht recht erhebend finden mag?

Die Kläger in Braunfeld sind Einzelhändler in Philadelphia, die in dem Sonntagsverkaufsverbot des Staates Pennsylvania nicht nur, wie bereits in McGowan verhandelt, eine Verletzung der Trennung von Staat und Kirche sehen, sondern zusätzlich geltend machen, in ihrer eigenen Religionsausübung, in ihrem free exercise of religion beeinträchtigt zu sein. Die Ladenbesitzer sind nämlich orthodoxe Juden, die nach ihren religiösen Gesetzen vom Sonnenuntergang am Freitag bis zum Sonnenuntergang am Samstag Abend Sabbat halten. Folgten sie diesem Sabbatsgebot und zugleich den staatlichen

44 BVerfG (Fn. 8), Rd. 109.

$45 \mathrm{Zu}$ ihnen gehört auch Stadtmauer, der sie als Beleg für ,the lesser status of Jews, Muslims, and other non-Christians" in den USA hält, ders. (Fn. 41), S. 213.

46 Benannt nach der grundlegenden Entscheidung Sloan v. Lemon, 413 U.S. 825 (1973). Zur wechselhaften Anwendung dieses Tests in der Praxis des Gerichts und den zahlreichen konkurrierenden Theorien einzelner Richter siehe Walter (Fn. 14), S. 132 ff. und Rotunda / Nowak, Treatise (Fn. 15), S. $453 \mathrm{ff}$.

47 Berger, The Less We Emphasize the Christian Religion, the Further We Fall into the Abyss of Poor Character and Chaos, Thurgood Marshall Law Review 22 (1997), S. 233 ff., S. 246.

48 Braunfeld v. Brown (Fn. 9).

49 In dem wieder vollständig auf die Ausführungen in Braunfeld verwiesen wird, Gallagher v. Crown Kosher Market (Fn. 9), S. 631. 
Gesetzen, so ihr Argument, blieben ihnen effektiv nur viereinhalb Tage um ihr Geschäft zu betreiben: Ein erheblicher wirtschaftlicher Nachteil, der ihnen entstünde, nur weil sie ihrem Glauben folgten.

Der U.S. Supreme Court hält gleich zu Beginn fest, dass das streitgegenständliche Gesetz keine religiösen Praktiken verbiete, sondern es reguliere lediglich ,a secular activity and, as applied to appellants, operates so as to make the practice of their religious beliefs more expensive. "50 Eine solche Verteuerung der Religionsausübung sei indes keine Verletzung der grundrechtlichen Freiheit der Kläger. Eine solche lediglich indirekte Beschwer könne nicht dazu führen, die Gestaltungsfreiheit des Gesetzgebers zu beschneiden: ${ }^{51}$

„, [If] the State regulates conduct by enacting a general law within its power, the purpose and effect of which is to advance the State's secular goals, the statute is valid despite its indirect burden on religious observance unless the State may accomplish its purpose by means which do not impose such a burden. "52

Die Entscheidung des U.S. Supreme Court in Braunfeld, jüdischen Gewerbetreibenden keine Ausnahme vom sonntäglichen Ladenschluss zu gewähren, reiht sich ein in die Tradition der free-exercise-Rechtsprechung des Gerichts, deren zentrale Frage ist, ob religiöse Praktiken Ausnahmen von allgemeinen gesetzlichen Anordnungen (generally applicable laws) rechtfertigen oder gar erforderlich machen. ${ }^{53}$ Die (moderne) Geschichte dieser Rechtsprechung ist die Geschichte dieses immer wiederkehrenden Streits um solche religiös motivierten Ausnahmen von allgemeinen Verbotstatbeständen (sog. $a c$ commodations), Ausnahmen etwa vom Polygamieverbot für Mormonen, ${ }^{54}$ von der Schulpflicht für die Amish People ${ }^{55}$ oder von Drogenverboten für Zeremonien indigener Religionen $^{56}$ - um nur ein paar wenige der berühmtesten Fälle zu zitieren. ${ }^{57}$

50 Braunfeld v. Brown (Fn. 9), S. 605.

51 Ebd.

52 Braunfeld v. Brown (Fn. 9), S. 607.

53 Vgl. Hamilton, God vs. the Gavel: Religion and the Rule of Law, 2005, S. 274: „The hard question that has been at the heart of the religious debate since the $1960 \mathrm{~s}$ is when, if ever, a religious individual or institution should be given freedom from the general law".

54 Eine solche verneinend: Reynolds v. United States, 98 U.S. 145 (1878) (nicht unter http:// www.findlaw.com abrufbar).

55 Diese gewährend: Wisconsin v. Yoder, 406 U.S. 205 (1972).

56 Wiederum verneinend: Employment Div., Ore. Dept. of Human Res. v. Smith, 494 U.S. 872 (1990).

57 Eine weitere, nicht weniger klassische Entscheidung weist einen indirekten Bezug zur Sonntagsruhe auf: so waren der Klägerin in Sherbert v. Verner (Fn. 9), einer den Samstag heilig haltenden Siebenten-Tags-Adventistin, vom Staat South Carolina Arbeitslosenhilfe versagt worden, nachdem ihr aufgrund ihrer Weigerung, samstags zu arbeiten, gekündigt worden war. Ihre Arbeitslosigkeit beruhte daher, nach Ansicht der Sozialverwaltung des Bundesstaates, auf ihrer Weigerung, passende Arbeit anzunehmen. Der U.S. Supreme Court sah durch diese Entscheidung die Klägerin in der free exercise ihrer Religion verletzt, da sie so faktisch zwischen ihrer Religionsausübung und den staatlichen Leistungen wählen müsse. Dies käme einem Bußgeld für ihre religiöse Observanz gleich: „Governmental imposition of such a choice puts the same kind of burden upon the free exercise of religion as would a fine imposed against appellant for her Saturday worship“ (ebd., 404). Den entscheidenden Unterschied zu Braunfeld sieht die Mehrheit des Gerichts in den Alternativen des Gesetzgeber: die Zwecke 
Ein weiteres Mal sieht der Supreme Court of Canada in seiner Entscheidung Edwards Books ${ }^{58}$ gerade den entscheidenden Punkt, nämlich ob die Religionsfreiheit Andersgläubiger überhaupt betroffen ist, anders. Wie oben schon angesprochen wurde, untersucht auch der Supreme Court of Canada zunächst die Frage, ob die angegriffene Sonntagsschutzgesetzgebung einen religiösen Zweck verfolgt und damit die freedom of religion der charter verletzt - in Big M Drug Mart hatte es den Lord's Day Act aus diesem Grund als verfassungswidrig verworfen. Anders in Edwards Books. Hier steht der Retail Business Holidays Act der Provinz Ontario aus dem Jahr 1983 auf dem verfassungsrechtlichen Prüfstand. Anhand desselben Maßstabes erkennt das Gericht hier, das Gesetz sei „enacted with the intent of providing uniform holidays to retail workers“ und sei kein ,surreptitious attempt to encourage religious worship“ .59

der Arbeitslosenversicherung ließen sich sehr wohl mit einer Rücksichtnahme auf Sabbatianer verwirklichen, diejenigen eines allgemeinen Ruhetages aber nicht. Implizit liegt dieser Rechtssprechung somit ein Gedanke der Verhältnismäßigkeit zugrunde. Der feine Unterschied zu Braunfeld liegt tatsächlich darin, dass die dort von den Klägern erlittenen wirtschaftlichen Einbußen indirekte Folge der gesetzlichen Sonntagsruhe, der Verlust der Leistungen in Sherbert aber direkte Folge der eigenen Samstagsruhe ist, Walter (Fn. 14), S. 312 f. Die beiden Entscheidung für unvereinbar hielt aber Justice Stewart in seinem dissent, Sherbert v. Verner (Fn. 9), 417 f. Interessant erscheint aber vor allem der Zusammenhang mit einer weiteren Entscheidung, die wenn auch nicht den Sonntag, so doch die Praxis religiöser Ruhetage im Arbeitsleben betrifft: Estate of Thornton v. Caldor, Inc. (Fn. 9). Dort stand eine Kündigungsschutzvorschrift des Staats Connecticut auf dem Prüfstand. Der Kläger hatte sich nachdem er wegen seiner Weigerung sonntags zu arbeiten - die Sunday closing laws waren hier schon 1977 weitgehend gefallen (ebd., Fn. 2) - auf diese Vorschrift berufen: „An employee's refusal to work on his Sabbath shall not constitute grounds for his dismissal" (ebd., S. 706). In seiner äußerst knappen Entscheidung erklärt der U.S. Supreme Court, die Vorschrift verstoße gegen no establishment, da sie gegenüber anderen Interessen des Arbeitgebers und der anderen Arbeitnehmer dem religiösen Interesse des einen absoluten Vorrang gewähre. Zum einen exemplifizieren Sherbert und Thornton so die innere Spannung des US-amerikanischen Religionsverfassungsrechts zwischen no establishment und free exercise, den ,natural antagonism between a command not to establish religion and a command not to inhibit its practice“" (Rotunda / Nowak, Treatise (Fn. 15), S. 446). Der Staat darf eine religiös motivierte Weigerung, an einem bestimmten Tag erwerbstätig zu sein, nicht mit der Kürzung von Sozialversicherungsleistungen bedrohen; er ist aber zugleich ebenso daran gehindert, zu verordnen, eine solche Weigerung dürfe keine arbeitsrechtlichen Folgen haben. Darin äußert sich aber zum anderen auch - ohne das dies in den Urteilen explizit wäre - eine Beschränkung auf die Staatsgerichtetheit der Grundrechte: Das öffentliche Recht (das Ordnungsrecht wie die Leistungsverwaltung) müssen so eingerichtet werden, dass sie Religionsausübung nicht behindern (Sherbert: free exercise); der Staat kann aber den Rechtsverkehr nicht so gestalten, dass der Religionsausübung absoluter Vorrang gebührt (Thornton: no establishment). Im Rahmen eines Verfassungsrechtsverständnisses, das Grundrechte auch als Wertentscheidungen für die gesamte Rechtsordnung versteht, die aufs Privatrecht Drittwirkung ausüben, wäre die Frage der Zulässigkeit eines Verbotes, Arbeitnehmer wegen religiös motivierter Arbeitsweigerung an bestimmten Tagen zu entlassen, mit einiger Wahrscheinlichkeit anders entschieden worden.

58 R. v. Edwards Books and Art Ltd. (Fn. 9).

59 R. v. Edwards Books and Art Ltd. (Fn. 9), Rd. 62. Das Gericht geht insbesondere wegen der Einbeziehung nichtchristlicher Feiertage wie Victoria Day, Canada Day oder Labour Day von nichtreligiösen Zwecken des Gesetzgebers aus. 
Aber der Supreme Court of Canada fragt in Edwards Books über die Zwecke des Gesetzes hinaus nach dessen Effekt. ${ }^{60}$ Unter detaillierter Auseinandersetzung mit Braunfeld kommt das Gericht dabei zum gegenteiligen Schluss wie der U.S. Supreme Court: In beinahe wettbewerbsrechtlichem Ton vergleicht es die Chancen derjenigen, deren Religion den Sonntag als Ruhetag vorschreibt, mit den Chancen derer, die den Samstag heiligen. Die wirtschaftlichen Einbußen der letzteren hält es für substanziell genug, als indirekte Belastung die Religionsfreiheit verkürzen. ${ }^{61}$

$* * *$

Dass die Verallgemeinerung des christlichen Ruhetages zum allgemeinen, für alle gültigen Ordnungsrecht ein Problem für die Nicht-Christen darstellen könnte, möglicherweise sogar deren Religionsfreiheit einschränkt, entgeht dem Bundesverfassungsgericht vollständig. In den nordamerikanischen Fällen ist der Sonntag das Diktat der Mehrheit, des demokratischen Gesetzgebers, und damit notwendigerweise verfassungsrechtlich auch ein Problem der Rechte von Minderheiten, denen durch die Kumulation des staatlichen Ordnungsrechts und der eigenen Überzeugungen empfindliche Nachteile entstehen. Säkulare Wohlfahrtsgesetzgebung im Konflikt mit religiösen Praktiken lautet das Problem, für das die Gerichte in Braunfeld und Edwards Books unterschiedlich differenzierte Lösungen anbieten.

Man sollte meinen, dass dieser Konflikt zwischen der Sonntagsschutzgesetzgebung und den Interessen der nicht-christlichen Bevölkerung im Kontext des deutschen Verfassungsrechts noch verschärft ist: Denn aus Sicht der amerikanischen Gerichte steht ja „nur" der moderne sozialpolitische Wille gegen die religiösen Rituale Einzelner. Aus der Sicht des deutschen Verfassungsrechts aber stellt sich der Staat mit dem sonntäglichen Ladenschluss schützend vor die Religionsausübung der Christen, indem er deren religiöse Praxis der Sonntagsruhe gesetzlich verordnet. Die nichtchristliche bzw. anders- oder nichtreligiöse Bevölkerung ist somit verpflichtet einer christlichen Praxis zu folgen. Das Minderheitenproblem aus Braunfeld und Edwards Books wird somit erst unter dem deutschen Verfassungsrecht eklatant: denn hier sind jüdische Ladenbesitzer nicht deshalb verpflichtet (auch) am Sonntag zu schließen, weil der Gesetzgeber diesen gemeinsamen Tag der Ruhe aus Gründen der allgemeinen Erholung und der Synchronisation außerökonomischer sozialer Kontakte für geboten hält; sie sind dazu verpflichtet, weil der Schutz christlicher Religionsausübung dies erfordert.

Im Raum steht somit eigentlich explizit die Verallgemeinerung, ja Oktroyierung fremder, nicht geteilter Glaubensinhalte, die sowohl der U.S. Supreme Court als auch der Supreme Court of Canada mit ziemlicher Sicherheit als Verletzung der Religions-

60 Die Dogmatik des no establishment war wie ausgeführt worden ist zum Zeitpunkt von $M c$ Gowan noch nicht über einen reinen purpose test hinaus gekommen.

61 Das Gericht verwirft das Gesetz im Ergebnis dennoch nicht, weil es die im Gesetz selbst vorgesehenen Ausnahmemöglichkeiten für kleinere und Familienbetriebe für ausreichend hält, R. v. Edwards Books and Art Ltd. (Fn. 9), Rd. 121 ff. Das Gericht prüft also anders als der U.S. Supreme Court wie das Bundesverfassungsgericht Grundrechtsverstöße zweistufig, nach Eingriff und Eingriffsrechtfertigung. 
freiheit von Nicht-Christen bewerten würden. ${ }^{62}$ Sie vermeiden diese Konsequenz indem sie den Gesetzen eine säkulare Finalität attestieren und indirekte Belastungen für hinnehmbar erklären (Braunfeld) bzw. durch bestehende Ausnahmetatbestände für hinreichend abgefedert halten (Edwards Books). Das Bundesverfassungsgericht will aber auf das Gegenteil hinaus, nämlich auf die religiöse Finalität des Sonntagsschutzes. Gerade weil das Bundesverfassungsgericht feststellt, der Sonntagsschutz diene dem Schutz der christlichen Religionsausübung, hätte sich ihm eigentlich die in einer Allgemeinverbindlicherklärung eines Glaubensinhaltes angelegte Problematik für diejenigen, die diesen Glauben nicht teilen, aufdrängen müssen. Aus der Sicht von Braunfeld und Edwards Books erscheint ganz unverständlich, wie die Gewährleistungen des Art. 139 WRV nicht in einem (verfassungsimmanenten) Spannungsverhältnis zu den Gewährleistungen des Art. 4 Abs. 1 und 2 GG stehen können. Nichts hierzu im Sonntagsurteil.

Im Gegenteil: Für das Bundesverfassungsgericht ist ja die Sonntagsruhe aller ein Recht einer Minderheit! Ein Recht der Minderheit zum einen verfassungstheoretisch gesehen, denn die Verteidigung des gesetzlichen Sonntagsschutzes gegen den liberalisierenden Gesetzgeber auf dem Rechtswege ist nach dem Sonntagsurteil eine grundrechtliche Gewährleistung. In Berlin ist das Recht der Christen auf Arbeitsruhe am Sonntag indes auch faktisch ein Recht einer Minderheit: dort sind nämlich nur etwas mehr als ein Drittel aller Bewohner Mitglieder einer Kirche. An einem durchschnittlichen Sonntag im Jahr 2008 besuchten 1,82 \% aller Berliner einen Gottesdienst. ${ }^{63}$

Wie kann man das verständlich machen? Etwa jemandem, der in der verfassungsrechtlichen Gedankenwelt von Braunfeld lebt?

Dass sich die Religionsfreiheit nicht darin erschöpft, den Christen zu garantieren, dass sie ihren heiligen Sonntag als Ruhetag begehen dürfen, sondern dass sie den Christen einen Anspruch darauf verleiht, dass die gesamte Bevölkerung an ihrem heiligen Ruhetag Ruhe hält, ist alles andere als selbstverständlich. Es ist schon nicht selbstverständlich, dass die Religionsfreiheit durch den Verzicht anderer auf einen Ruhetag überhaupt betroffen sein kann. Das Bundesverfassungsgericht führt insoweit aus, die Lockerung des Sonntagsverkaufsverbotes in Berlin führe zu wahrnehmbarer ,,werktäg-

62 Der Supreme Court of Canada setzt sich mit dieser Frage in Edwards Books ausdrücklich auseinander. Einen religiösen Zwang vermag er in dem Retail Act nicht erkennen, da dieser säkulare Ziele verfolge und damit nur zufällig (wie eben beispielsweise auch das Tötungsverbot) mit einer christlichen Glaubensvorschrift zusammen falle: „In my view, legislation with a secular inspiration does not abridge the freedom from conformity to religious dogma merely because statutory provisions coincide with the tenets of a religion.", R. v. Edwards Books and Art Ltd. (Fn. 9), Rd. 101.

63 Dies ergibt sich aus den Zahlen des Statistischen Jahrbuchs 2009 des Amtes für Statistik Berlin-Brandenburg (als Download erhältlich unter http://www.statistik-berlin-brandenburg.de). Danach sind 994.217 Berliner Mitglied einer der beiden großen Kirchen (675.779 der evangelischen und 318.438 der katholischen, a.a.O., Kapitel 04 - Bildung und Kultur, S. 174). Bei einer Gesamtbevölkerung von 3.431 .675 (a.a.O., Kapitel 01 - Gebiet und Bevölkerung, S. 33) entspricht dies einem Anteil von 34,1\%. Insgesamt 53.232 Berliner besuchten an einem durchschnittlichen Sonntag 2008 einen Gottesdienst (17.182 einen evangelischen und 36.104 einen katholischen, a.a.O., Kapitel 04 - Bildung und Kultur, S. 174). 
liche[r] Geschäftigkeit“ an den verkaufsoffenen Sonntagen und dies betreffe „,namentlich auch die Gläubigen christlicher Religionen und die Religionsgemeinschaften selbst, nach deren Verständnis der Tag ein solcher der Ruhe und der Besinnung" sei. ${ }^{64}$

Welcher Begriff von Freiheit liegt denn zugrunde, wenn Freiheit betroffen ist durch die Wahrnehmbarkeit der Geschäftigkeit anderer, und diese Betroffenheit sogar das Eingreifen der staatlichen Schutzmacht erforderlich macht? Welches Freiheitsverständnis liegt zugrunde, wenn Teil einer Freiheitsgewährleistung das durchsetzbare Recht ist, allen anderen wahrnehmbare Geschäftigkeit zu untersagen? ${ }^{65}$ Eine wirkliche Auseinandersetzung mit diesen Fragen findet im Sonntagsurteil nicht statt, und die dogmatische Begründung des Urteils bleibt daher wenig überzeugend.

Um den sonntäglichen Ladenschluss mit seinem Urteil stärken zu können, braucht das Gericht eine subjektive Rechtsstellung der klagenden Kirchen. Es findet diese in Art. 4 GG und der aus ihr abgeleiteten ,Schutzpflicht“ des Staates, deren Gehalt durch die Kirchenartikel näher bestimmt werde. Dies ergebe sich aus der ,funktionale[n] Ausrichtung der [...] Kirchenartikel auf die Inanspruchnahme des Grundrechts aus Art. 4 Abs. 1 und 2 GG“, die aus dem „Religionsbezug des Art. $139 \mathrm{WRV}^{\text {“ }}$ gefolgert wird. ${ }^{66}$ Der „Religionsbezug“ des Art. 140 GG und der durch ihn inkorporierten Weimarer Kirchenartikel ist allerdings vollkommen offensichtlich und auch vollständig unkontrovers. Ihn historisch und systematisch herzuleiten wirkt wie ein Scheingefecht, das das Nichtstattfinden eines anderen verdeckt: Man vermisst im Sonntagsurteil nämlich jegliche inhaltliche Erläuterung, weshalb der Religionsbezug des Art. 139 WRV gerade ein Bezug auf die grundrechtliche Religionsfreiheit ist, warum der gesetzliche Schutz des Sonntages als arbeitsfreiem Tag für alle ein Teil der unverletzlichen „Freiheit des Glaubens, des Gewissens und [der] Freiheit des religiösen und weltanschaulichen Bekenntnisses“ sowie der „ungestörte[n] Religionsausübung“ der Christen ist. Oder ein Teil des staatlichen Schutzes dieser Freiheit. Der Gedanke, eine sonntägliche Ladenöffnung in Berlin betreffe diese Freiheit der Christen und ihrer Kirchen, auch wenn sie selbst weder ein- noch verkaufen, ist recht anspruchsvoll. Die dadurch aufgeworfenen grundrechtstheoretischen Fragen werden im Sonntagsurteil vom grundrechtsdogmatisch-technischen Vokabular „funktionale Ausrichtung“ und „Schutzpflicht“ nur verschleiert. ${ }^{67}$

Angesichts des Sonntagsurteils gibt es somit keinen Anlass, die Besorgnis Martin Heckels, eine „Vergrundrechtlichung“ der staatskirchenrechtlichen Gewährleistungen könne eine „laizistische oder individualistische Verkürzung der Religionsfreiheit“ be-

64 BVerfG (Fn. 8), Rd. 165.

65 Interessant wiederum die praktisch gegenteilige Annahme des U.S. Supreme Court in Estate of Thornton v. Caldor, Inc. (Fn. 9), die Rücksichtnahme auf den Ruhetag anderer bedeute „significant burdens on other employees“ (ebd., S. 710).

66 BVerfG (Fn. 8), Rd. 139.

67 Vosgerau hat die vom Bundesverfassungsgericht in seinen Abtreibungsentscheidungen gefundene Schutzfunktion der Grundrechte als einen „Gemeinwohlvorbehalt" interpretiert, der lediglich ,individualistisch“ verpackt worden sei, ders., Schutzlos allein, Frankfurter Allgemeine Zeitung vom 11. März 2010, S. 8. Dann stünden sich im Falle der hier diskutierten Urteile keine unterschiedlichen Auffassungen von Freiheit gegenüber, sondern ein Gericht, das die Problematik des Sonntagsschutzes vom Gemeinwohl her denkt und eines, das von den Freiheitsrechten des Einzelnen ausgeht. 
wirken, ${ }^{68}$ zu teilen. Die Wandlung des Art. 139 WRV zu einem Gewährleistungsgehalt der grundrechtlichen Religionsfreiheit der christlichen Kirchen durch das Sonntagsurteil hat vielmehr den gegenteiligen Effekt: es manifestiert das, was Heckel die „Freiheit des Wirkens in der Welt" der Religionsgemeinschaften nennt: ${ }^{69}$ Die Kirchen haben nun das einklagbare Recht, allen anderen den Einzelhandel am Sonntag zu verbieten. Ihre Freiheit aus Art. 4 GG ist politische Gestaltungsmacht. Diese Freiheit unterscheidet sich damit fundamental von derjenigen aus free exercise, die allenfalls politische Gestaltungsmacht beschränken kann.

Vor dem Sonntagsurteil hatte das Bundesverfassungsgericht bekanntlich eine subjektivrechtliche Dimension des Sonntagsschutzes ausdrücklich verneint: „Art. 139 WRV enthält lediglich eine objektivrechtliche Institutsgarantie ohne subjektive Berechtigung“. ${ }^{70}$ Das Bundesverfassungsgericht möchte sein Sonntagsurteil indes nicht als Abweichung von seiner bisherigen Rechtsprechung gelten lassen: Offen geblieben sei in dem eben zitierten Beschluss nämlich,

„ob und inwieweit gerade Art. 139 WRV im Zusammenwirken mit Art. 4 Abs. 1 und 2 GG oder anderen Grundrechten Religionsgemeinschaften oder anderen Betroffenen eine Durchsetzung des Sonn- und Feiertagsschutzes ermöglicht. "71

Diese Lesart der eigenen älteren Rechtsprechung vermag nicht zu überzeugen. Denn der Beschwerdeführer im Buß- und Bettagsverfahren hatte sich ausdrücklich auf eine Verletzung seiner Religionsfreiheit berufen. Und das Bundesverfassungsgericht hatte ihm beschieden:

„Die Verbürgungen des Art. 4 I und II GG sind [durch die Streichung des Buß- und Bettages als staatlich anerkannter Feiertag] nicht verletzt. [...] Aus Art. 4 I und II GG folgt kein Anspruch des einzelnen gegen den Staat, bestimmte kirchliche Feiertage ganztägig als Tage der Arbeitsruhe auszuweisen " . 72

Damit war eindeutig eine subjektiv-rechtliche Dimension des Art. 138 WRV sowohl „aus sich selbst“, als auch ,in Verbindung mit“ Art. 4 GG verneint worden. So sahen es auch die meisten in der Literatur. ${ }^{73}$

$* * *$

68 Heckel, Zur Zukunftsfähigkeit des deutschen „Staatskirchenrechts“ oder „Religionsverfassungsrecht"?, Archiv des öffentlichen Rechts 134 (2009), S. 309 ff., $330 \mathrm{ff}$.

69 Heckel (Fn. 68), S. 331 (Hervorhebung im Original).

70 BVerfG, Neue Juristische Wochenschrift 1995, S. 3378 ff., S. 3379 (Streichung des Buß- und Bettages als staatlich anerkannter Feiertag).

71 BVerfG (Fn. 8), Rd. 121.

72 BVerfG (Fn. 70), S. 3378.

73 Kästner, Der Sonntag und die kirchlichen Feiertage, in Listl / Pirson (Hrsg.), Handbuch des Staatskirchenrechts, Bd. II, 2. Aufl. 1995, § 51, S. 340 f.; Korioth (Fn. 12), Rd. 4 und 42; Ehlers in Sachs (Hrsg.), GG, 4. Aufl. 2007, Art. 140 GG, Art. 138 WRV, Rd. 1; Mattner, Sonntagsruhe im Spiegel des Grundgesetzes und der Feiertagsgesetze der Länder, Neue Juristische Wochenschrift 1988, S. 2207 ff., S. 2207 f.; de Wall, Zum subjektiven Recht der Kirchen auf den Sonntagsschutz, Neue Zeitschrift für Verwaltungsrecht 2000, S. 857 ff., 
Die Konsequenz dieser Verortung der Sonntagsschutzgarantie des Art. 139 WRV in den Grundrechten ist ein Gleichbehandlungsproblem, das wiederum dem amerikanischen komplementär ist. Dort stellt sich die Frage, ob Einzelne aus der allen verordneten Sonntagsruhe ausscheren dürfen. Hier stellt sich nunmehr sie Frage, ob dem grundrechtlichen Anspruch der Christen nicht eine entsprechende Gewährleistung für andere Religionen folgen muss. ${ }^{74}$

Es gibt im Sonntagsurteil indes keinen Hinweis darauf, dass das Bundesverfassungsgericht einen solchen „Ausgleichsanspruch“ auf gesetzliche Ruhe- und Feiertage für andere Religionen für verfassungsrechtlich verbürgt hält. Vielmehr hält das Gericht fest:

\begin{abstract}
„, Die Pflicht des Staates zu weltanschaulich-religiöser Neutralität steht einer Konkretisierung des Schutzgehalts des Art. 4 Abs. 1 und 2 GG durch Art. 139 WRV nicht entgegen. Denn die Verfassung selbst unterstellt den Sonntag und die Feiertage, soweit sie staatlich anerkannt sind, einem besonderen staatlichen Schutzauftrag und nimmt damit eine Wertung vor, die auch in der christlich-abendländischen Tradition wurzelt und kalendarisch an diese anknüpft. "75
\end{abstract}

Das Gericht geht daher offenbar selbst nicht davon aus, dass die Religionsfreiheit aus Art. 4 GG den nichtchristlichen Religionen eine dem Art. 139 WRV entsprechenden Anspruch gewährleistet. Sonst müsste es dieses Christenrecht nicht vor der weltanschaulich-religiösen Neutralität des Staates rechtfertigen. Es mag stimmen, dass das Grundgesetz in Anknüpfung an christlich-abendländische Tradition die gesetzliche Verankerung des christlichen Sonntags unter Bestandsschutz stellt ohne anderen Religionen Vergleichbares zu liefern. Dann ist das Grundgesetz aber insoweit nicht neutral.

\title{
IV. Zuletzt: Der Sonntag im Verfassungstext
}

Dass der Sonntagsschutz im deutschen Verfassungsrecht etwas ganz anderes ist als im US-amerikanischen (oder dem kanadischen), dass das Bundesverfassungsgericht im Hinblick auf den Sonntagsschutz ganz andere Antworten gibt und vor allem ganz andere Fragen stellt - so könnte man zuletzt einwerfen - ist doch so verwunderlich nicht! Unter der ganzen Suche nach unterschiedlichen Themen und Zwecken, Ansätzen und Sichtweisen scheint eines fast vergessen worden zu sein: Er steht doch nun mal drin, der Sonntagsschutz! In der Verfassung wortwörtlich: „Der Sonntag und die staatlich anerkannten Feiertage bleiben als Tage der Arbeitsruhe und der seelischen Erhebung gesetzlich geschützt".

S. 859 f; Stollmann, Zum subjektivrechtlichen Gehalt des Art. 140 GG / 139 WRV, Verwaltungsarchiv 96 (2005), S. 348 ff. Von einer subjektivrechtlichen Dimension des Art. 139 WRV ging insbesondere Morlok aus, in Dreier (Hrsg.), Grundgesetz-Kommentar, Bd. III, 2. Aufl. 2008, Art. 139 WRV, Rd. 17 ff.; ders. / Heinig, Feiertag! Freier Tag? Die Garantie des Sonn- und Feiertagsschutzes als subjektives Recht im Lichte des Art. 139 WRV, Neue Zeitschrift für Verwaltungsrecht 2001, S. 846 ff., S. 848 ff.

74 Diese Konsequenz fordert bereits Classen (Fn. 39), S. 146.

75 BVerfG (Fn. 8), Rd. 121. 
Der Sonntag steht aber auch in der Verfassung der Vereinigten Staaten. An recht versteckter Stelle zwar, in Klammern noch dazu, aber dennoch: Gemäß Art. 1 Absatz 7 der United States Constitution muss jedes Gesetz, nachdem es vom Repräsentantenhaus und dem Senat beschlossen worden ist, um rechtskräftig zu werden, vom Präsidenten unterzeichnet werden. Das gibt dem Präsidenten die gefürchtete Waffe eines Veto in die Hand, das nur noch von Zweidrittelmehrheiten beider Häuser zu Fall gebracht werden kann. Doch beliebig lange Zeit hat der Präsident nicht, sich zu überlegen, ob er zeichnen soll oder nicht, denn - und hier kommt es endlich -

„If any Bill shall not be returned by the President within ten Days (Sundays excepted) after it shall have been presented to him, the Same shall be a Law, in like Manner as if he had signed it". ${ }^{76}$

Es steht somit fest, dass wenigstens der Präsident der Vereinigten Staaten am Sonntag die Arbeit ruhen lässt - jedenfalls die Arbeit am Veto. Aber in dieser unscheinbaren Bestimmung zur Fristberechnung steckt doch noch etwas mehr: denn die Wörter „(Sundays excepted)“ haben überraschenderweise einen ganz direkten Bezug zu den hier verhandelten bundesstaatlichen Sunday closing laws. Das nämlich weist Jaynie Randall in ihrer historischen Untersuchung zu der Vorschrift nach: ${ }^{77}$

Randall analysiert, die Verfassungsväter hätten mit der Zehn-Tagesfrist dem Präsidenten ausreichend Zeit zur gründlichen politischen Abwägung seines Vetorechts geben wollen, und ihm daher letztlich eine im Vergleich zu entsprechenden Regelungen in den bundesstaatlichen Verfassungen relativ lange Abwägungsfrist eingeräumt. ${ }^{78}$ Dieses die Vetoregelung tragende deliberation principle sei weiterhin der Grund dafür gewesen, dem Präsidenten in jedem Fall die Möglichkeit zu geben, die gesamten zehn Tage für die Abwägung über ein Veto auszunutzen. Eine solche Abwägung des Präsidenten sei nun aber keine einsame Überlegung einer einzelnen Person, sondern erfordere Beratung, also die Arbeit nicht nur des Präsidenten, sondern auch seiner Berater und Mitarbeiter, und verlangte von diesen vor allem auch - im späten 18. Jahrhundert - Reisen. ${ }^{79}$ Eben das Reisen verboten aber die Sonntagsschutzgesetze der Bundesstaaten mit einiger Konsequenz. In der Tat konnte Präsident Washington selbst einer Verhaftung bei einer sonntäglichen Durchreise durch Massachusetts nur knapp entgehen! ${ }^{80}$ Der Bundesverfassungsgeber sah sich also vor die Entscheidung gestellt, entweder den Präsidenten und dessen Berater und deren staff, sowie (im Falle eines sonntäglichen return of the veto) die Mitarbeiter des Kongresses, in Konflikt mit dem Gesetz zu bringen oder aber den Abwägungszeitraum des Präsidenten um einen oder zwei Tage zu beschneiden. Man entschied sich daher mit der Herausnahme des Sonntags aus der Berechnung der ZehnTagesfrist zugleich für eine gründliche Deliberation des Präsidenten und den Respekt gegenüber dem bundestaatlichen Ordnungsrecht. ${ }^{81}$ So hat also der gesetzliche Sonntagsschutz auch in der amerikanischen Verfassung eine Spur hinterlassen.

76 Hervorhebung von mir.

77 Randall, Sundays Excepted, Alabama Law Review 59 (2008), S. 507 ff.

78 Randall (Fn. 77), S. $512 \mathrm{ff}$.

79 Randall (Fn. 77), S. $521 \mathrm{ff}$.

80 Randall (Fn. 77), S. 526 und Fn. 143.

81 Randall (Fn. 77), S. 529ff. 
Dieser kleine Textteil der Verfassung, diese Spur auf die bundesstaatlichen Sunday closing laws, hat nichtsdestoweniger schon als Beleg für eine mächtige These herhalten müssen, nämlich derjenigen der USA als christlicher Nation, die einst einstimmig vom U.S. Supreme Court geteilt wurde. In Holy Trinity Church v. U.S. ${ }^{82}$ begründet das Gericht seine Entscheidung, Kirchen bei ihrer Priesterwahl eine Ausnahme vom allgemeinen Verbot, ausländische Arbeitskräfte anzuwerben, zu gewähren, mit eben jener überragenden Bedeutung des Christentums für die Rechts- und Gesellschaftsordnung der USA, und zitiert zum Beleg dessen ausufernd historisches Material, einfache Gesetze, bundesstaatliche Verfassungen und eben auch das „Sundays excepted“ aus Art. 1 Absatz 7 der United States Constitution:

"These, and many other matters which might be noticed, add a volume of unofficial declarations to the mass of organic utterances that this is a Christian nation." 83

Eine recht dünne Verfassungstextgrundlage für eine solch gewaltige Fundamentalthese. Man muss schon mit sehr leeren Händen dastehen, wenn man sich auf zwei Wörtchen zwischen zwei Klammern stürzt. Und größer könnte der Kontrast zum Bundesverfassungsgericht nicht sein, das, noch während es einen grundrechtlichen Schutz des christlichen Sonntags entdeckt, auf der Neutralität der Verfassungsordnung in Religionsfragen besteht.

Darin zeigen sich letztlich wohl die gänzlich unterschiedlichen religionsverfassungsrechtspolitischen Spannungen der beiden Verfassungsstaaten: Die eines gesellschaftlich in weiten Teilen zutiefst christlich geprägten Landes einerseits, indem die Begründung einer Christian nation im Gefüge seiner republikanischen Verfassung auf Wortklauberei angewiesen ist. Und diejenige einer Rechtsordnung andererseits, die sowohl verfassungsrechtlich als auch gesetzlich zahlreiche Privilegien für das Christentum bereitstellt, dies aber mit einer weltanschaulichen „Neutralität“ zu vereinbaren sucht, die den Bedürfnissen der postreligiösen Mehrheit und der andersreligiösen Minderheiten gerecht werden soll.

82143 U.S. 457 (1892) (nicht unter http://www.findlaw.com abrufbar).

83 Holy Trinity Church v. U.S. (Fn. 82), S. 471. 